\title{
Lexical Phenomenon in Linguistics Theories
}

\section{Muhammad Qodri}

Universitas Islam Negeri (UIN) Sulthan Thaha Saifuddin Jambi, Indonesia

e-mail : mqodri87@gmail.com

\begin{abstract}
Lexical is study of vocabulary and its meanings in one language or in a number of languages. Vocabulary is concerned mainly with derivation of words, structures, moral, syntactic connotations, idiomatic expressions, synonyms, and multiplicity of meanings. This research aimed to describe the Abstract lexical phenomenon in linguistic theories. The methodology used in this research was qualitative research method with the type of library research. The results of the research were the lexical theory is carried out in a number of procedures necessary to accomplish the lexicon of the language, acoustically, and not neglected widely.
\end{abstract}

Keyword Lexical Phenomenon; Linguistics Theory

$$
\begin{aligned}
& \text { المعجمية هي دراسة المفردات ومعانيها في لغة واحدة أو في عدد من اللغات. } \\
& \text { ويهتم علم المفردات من حيث الأساس باشتقاق الألفاظ، وأبنيتها، ودلالاتها } \\
& \text { المعنوياة والإعرابية، والتّعابير الإصطلاحية، والمترادفات وتعدد المعاني. ويهدف } \\
& \text { هذا البحث إلى وصف الظاهرة المعجمية في نظريات اللغوية. والمنهج } \\
& \text { المستخدم في هذا البحث هو منهج البحث الكيفي بنوع دراسـة مكتبية. أما } \\
& \text { نتائج هذا البحث هي: () النظرية المعجمية تقوم في عدد من الإجراءات } \\
& \text { الضرورية لإنجاز معجم اللغة تبدأ هذه الإجراءات بالجمع المعجمي اللازم }
\end{aligned}
$$

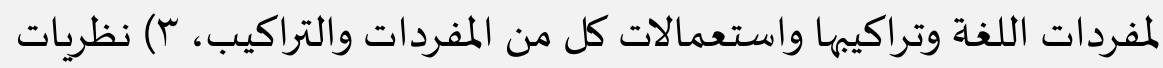

$$
\begin{aligned}
& \text { التصنيف المعجمي بتصنيف المادة التي يدرجونها في دائرة العربية المستعملة } \\
& \text { التي ليست محظورة صوتيا ولا مهملة استعمالا. }
\end{aligned}
$$
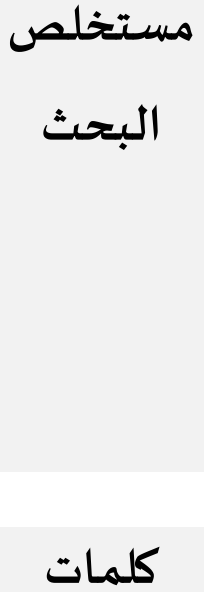

ظاهرة معجمية ؛ نظريات لغوية 


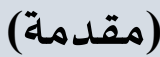

المعجم لله دور هام في محافظة اللغة خاصة اللغة العربية، بالمعجم تنتشر اللغة العربية إلى أي بلد كان. والمعجم هو كتاب يضم أكبر عدد من مفردات اللغـة مقرونة بشرح

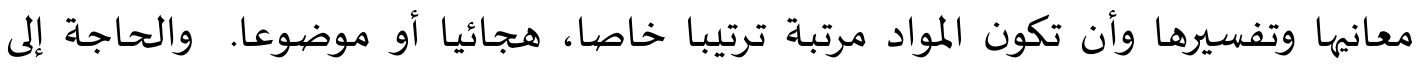
استخدام المعجم اللغوي حاجة ماسة ودائمة، بالنسبة إلى دارسي اللغة، فضلا عن أهميتها بالنسبة إلى كل من يتكلم اللغة، ذلك أن قدرة المتكلم على استيعاب المفردات محدودة بمـجال

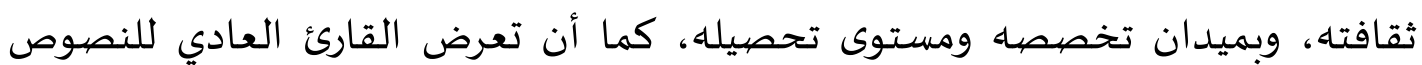
اللغوية في أي مجال، أمر يتكرر دائم(الغني 199:1919). والمعاجم المتوفرة هي المنتاجات الابتكارية لدى اللغويين والنتائج المحصولة في البحوث المعجمية. فبدى أن المعجمه هو من أمر مهم تعلماه لأناه يين معاني الكلمات تحليلية من حيث بيان شروطاه وظيفتاه وأهميتاه. أما الظاهرة المعجمية دلالية في جوهرها. وهذا ما يفيده تصنيف علم المين الدلالة إلى علم الدلالة معجمي وعلم دلالة تركيبي. كما يورثاه هذا مشكلات الدلالة المختلفة، وأبرزها تجريدية ظاهرة الدلالة، وعدم اقتصار الظاهرة على مستوى لغوي معين، فالدلالة صوتية وصرفية ونحوية وغياب البناء الكلي العام للظاهرة، وهو ما يريد أن يناقشه البحث بصفة رئيسية. يحاول الباحث أن يقوم بعرض النظرية المعجمية ونظريات الجمع والتصنيف والتركيب المعجمي، ويتناول هذا البحث على ظواهرالمعجمية.

جاء في لسان العرب "مادة عجمش: العُجم والعَجَمُ خلاف العُرب والعرب. والعُجْم جمع الأعجم الذي لا يفصح ولا يبين كلامه وإن كان عربي النسب، والأنثى عجماء. أما العجمي فهو الذي من جنس العجم أفصح أو لم يفصح، والأعجم الذي في لسـانه عجماة. وكل من لا قدر على الكلام فهو أعجم ومستعجم(منظور, 1907). واستعجم الرجل: سكت. واستعجمت عليه قراءته: انقطعت فلم يقدر على القراءة، المعجم : ديوان المفردات اللغة مرتب على حروف

$$
\text { المعجم. وحروف المعجم : حروف الهجاء. }
$$


عرّف اللغويون المعجم بأنه كتاب يضمر بين مفردات لغة ما ومعانيها واستعمالاتها في التركيب المختلفة، وكيفية نطقها، وكتابتها مع ترتيب هذه المفردات بصورة من صور الترتيب

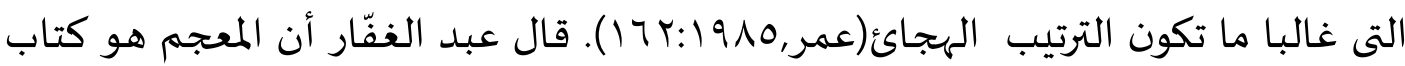
يضهمّ أكبر عدد من مفردات اللغة مقرونة بشرحها وتفسير معانيها على أن تكون الموادّ مرتبة

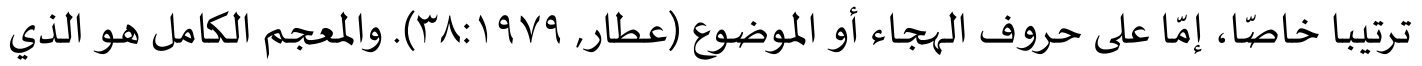
يضهم كل كلمة في اللغة مصحوبة يشرح معناها واشتقاقها وطريقة نطقها وشواهد تبين مواضع

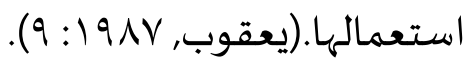

فالمعجم هو كتاب يحتوي مجموعاة من مفردات اللغة مرتبة ترتيبا أبجديا، أو في نظام أخر محدد مع شرح معانهها، وعادة ما يذكر المعلومات الخاصية بها في اللغة نفسها، أو في لغات أخرى، بالإضافة الم ذلك فإن القاموس تتعرض لطريقة نطقها، واشتقاقها والمتردفات، والإصطلاحات، مع ذكر الشواهد التوضيحة. ولابد لكل المعجم أن يكون مرتبا، لتسهيل القراء في بحث المفردات وفهمها وكذلك لاقتصار الوقت.

\section{أهمية المعجم وفوائده}

وأما أهمية المعجم وفوائده ما يلي :

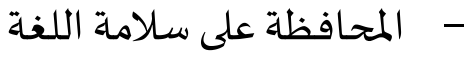

فاللغة العربية هي وعاء الثقافة وعنوان الهوية، والمحافظة عليها تعد محافظة على الذات والوجود ـ ويقول الأستاذ المرحوم مصطفى صادق الرافعي :وإذا كانت اللغة العربية بهذه المنزلة، كانت أمتها حريصة عليها، ناهضية بها، متسعة فيها، مكبرة لشأهها ... فأما إذا كان من شعبها التراخي والإهمال وترك اللغة للطبيعة السوقية وإصغار أمرها وتهوين خطرها، وإيثار غيرها بالحب والإكبار، فهذا شعب خادم لا مخدوم، تابع لا متبوع، ضعيف عن وهن تكاليف السيادة، من هنا أن المعجم لله دور هام في المحافظة على سلامة اللغة. - - جعل اللغة قادرة على مواكبة العلوم والفنون

إن اللغة العربية ليست ضيقة ولا عاجزة عن المواكبة لنتائج العلوم والفنون، بل هي صالحة للتدريس العلوم والفنون بسبب اتساع طاقتها الاستيعابية المعجمية بالمقارنة مع اللغات الأجنبية. ومن المزايا الإيجابية للغة العربية أهها تستفيد من ظاهرة الإعراب والتوليد والاشتقاق بكل أنواعاه و من ظاهرة التعريب والتعجيم، وكل هذا يساعدها على الانفتاح 
والاستفادة من كل لغات العالم. ويزيدها شرفا أن الله حمل القرآن المكتوب بالبيان العربي ولغته الرائعة كثيرا من الحقائق العلمية كالتي تتعلق بالأجنة والفلك والطبيعة. فكيف يعقل

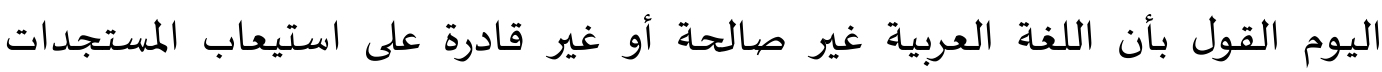

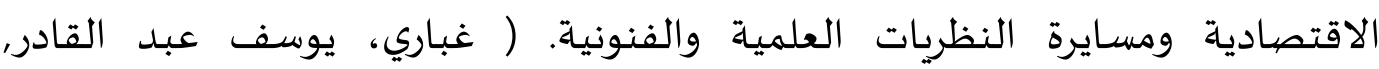

(1) $\varepsilon-11 r: Y . .9$

- - الكشف عن معاني الألفاظ المجهولة والغامضاة

الكشف في المعجم عن كلمة ؛ لتعرف على معانها المختلفة، و كيفية ضبط بنيتها،

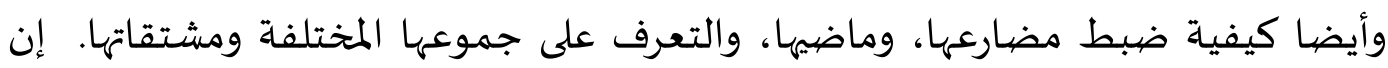
المعجم يكشف معني الألفاظ المجهولة والغامضية على سبيل :

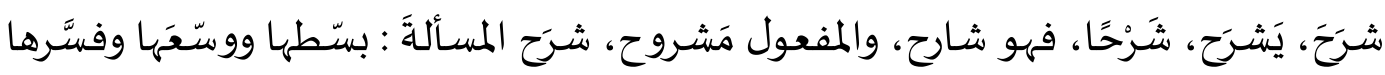

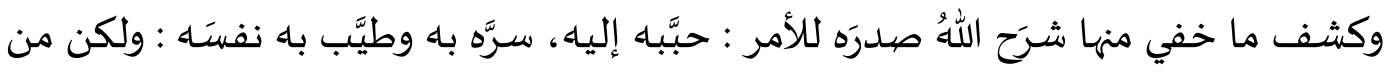
فتح صدره للكفر. - معرفة ظواهر اللغوية كالمشترك اللفظي والأضداد الظواهر اللغوية المشتركة في اللغة العربية هي المشترك اللفظي : اللفظ الواحد الدال

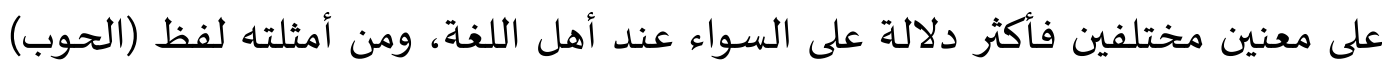

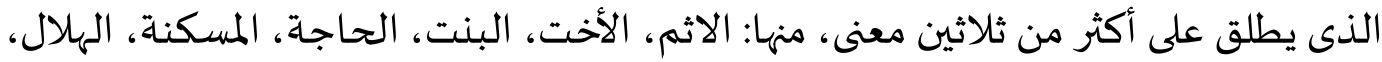

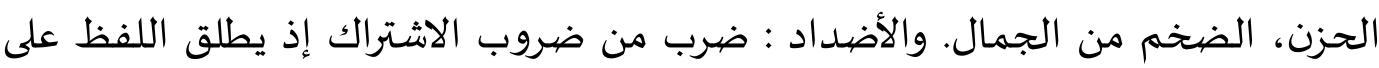

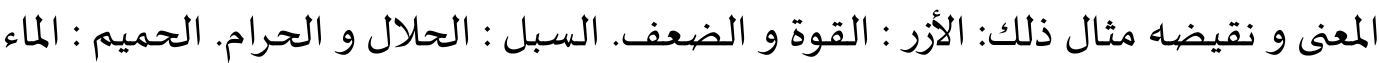

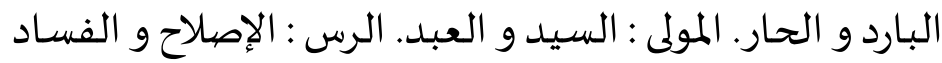
- - معرفة أصل اللفظ واستقاقاته

هو أن يؤخذ من لفظة ما كلمة أو أكثر مع التناسب في المعنى بين اللفظة المشتقة وما فأها

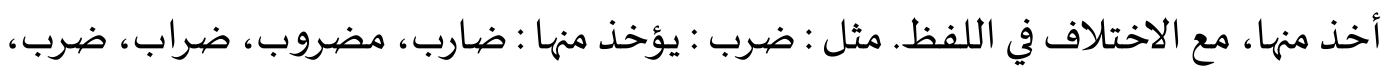

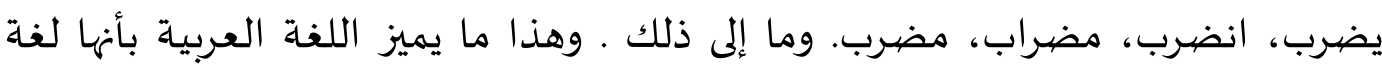

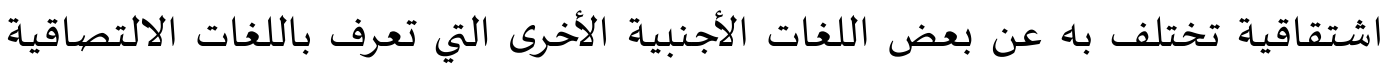

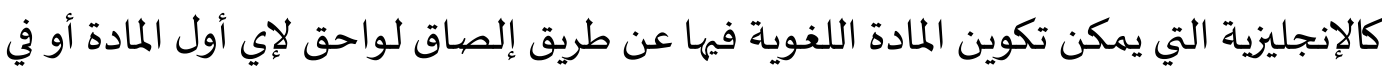
آخرها - معرفة كون اللفظة عامية أو فاصحة - معاصة 
لا شك أن اللغة الفصي و اللغة العامية مختلفة في كل مكان. نستخدم الكثير من الكلمات من العامية لكن لا توجد في اللغة الفصجي ـ يختلف النطق عن الحروف و الكلمات

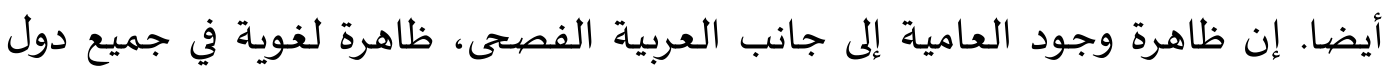
العالم، ولكل منهما مجالاتاه واستعمالاته، وتعرف اللهجة العامية بأنها طريقة الحديث التي يستخدمها السواد الأعظم من الناس، وتجري بها كافة تعاملاتهم الكلامياة، وهي عادة لغوية

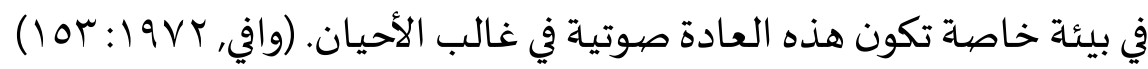

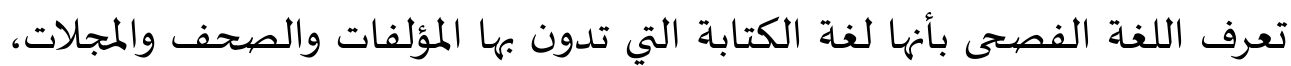
وشؤون القضاء والتشريع والإدارة، ويؤلف بها الشعر والنثر الفني، وتستخدم في الخطابة والتدريس والمحاضرات، وفي تفاهم العامة إذا كانوا بصدد موضوع يمت بصلة إلى الآداب والعلوم.(مجد البرازي, 1919 :00) وظيفة المعجم

هناك مجموعة من الوظائف يجب أن يؤديها المعجم وهي : - شرح الكلمة وبيان معناها أو معانها، إما في العصر الحديث فقط أو مع تتبع معناها أو معانيها عبر العصور. المثال : معجم المنور.

- بيان كيفية نطق الكلمة. المثال : معجم البريد الإلكترونيكي. - بيان كيفية كتابة الكلمة.

- تحديد الوظيفة الصرفية للكلمة. المثال : معجم الوسيط. - بيان درجة اللفظ في الاستعمال، ومستواه في سلم التنوعات اللهجية. المثال : معجم البريد الإلكترونيكي. - تحديد مكان النبر في الكلمة. والنبر باختصـار هو إعطاء بروز معين لأحد مقاطع الكلمة دون المقاطع الأخرى. ولما كان النبر في اللغة العربية الفصحي لايؤدي انتقاله من مقطع إلى مقطع

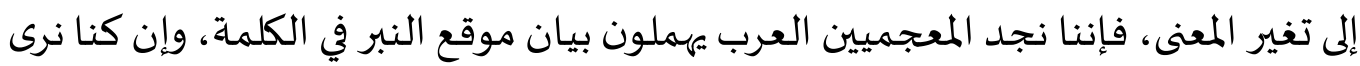
أن بيان موضعاه ضروري لمن يريد تحقيق النطق العببي الفصيح، كما أنه ضروري بالنسبة لمن يريد أن يتعلم كيفية النطق الحديث للهجات العربية.(عمر (910 19 : 77 (1).

إن النظرية المعجمياة تقوم في عدد من الإجراءات الضرورية لإنجاز معجمي اللغة تبدأ هذه الإجراءات بالجمع المعجمي اللازم لمفردات اللغة وتراكيبها واستعمالات كل من المفردات 
والتراكيب، ويتبع ذلك إجراءا الوصف والتصنيف للمادة المجموعاة، كما يلزم تمام العمل المعجمي أن يكون ثمة تحليل دلالي للمادة المعجمية يكشف عن الأنظمة الدلالية التي تحكمها،

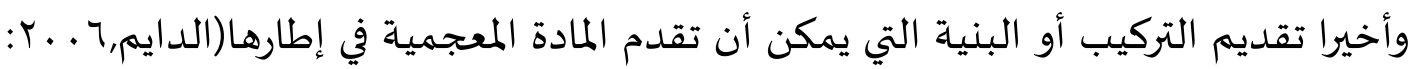

وقد انتقل المعنى المعجمي الى المجال الإصطلاحي، فصار التركيب عند اصطلاح

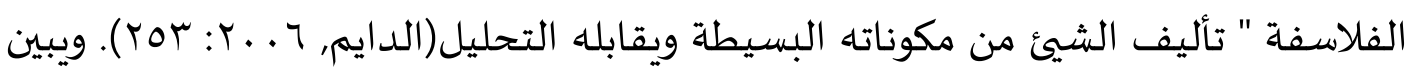
الشريف الجرجاني في تعريفاته اصطلاح التركيب في اللغة يقول "التركيب جمع الحرف البسيط ونظمه ليكون كلمة. التركيب يتطلب ثلاثة أمور تتمثل في وحدات صغرى، وعلاقات تقوم بـات بينها لتشكيل الأمر الثالث، وهو الوحدة الكبرى. وهذا ما يقوم في تراكيب اللغة المختلفة مثل التركيب الصهوتي الذي يتمثل في سمات صوتية تجتمع معا وفق قوانين محددة لتكوين الأصوات، وكذلك تتركب الكلمات معا وفق قوانين وعلاقات معينة لتشكيل وحدة الجملة الكبرى. اي لا يمكننا ان نتكلم عن تركيب مالم نملك وحدة نهائية كبرى ووحدات صغيرى

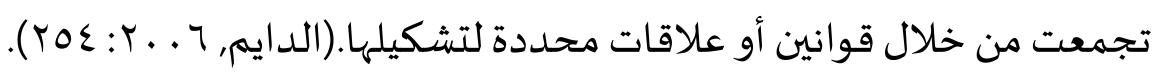
وقد قدم مفهوم النظام هذا فرديانند دي سوسير، وهو يمثل تفصيلا للمفهوم التراثي للتصريف لكنها يختلف في أنه يركز على العلاقات التي بين العناصر أكثر من العناصرير نفسها. ويعني ذلك أن النظام يتطلب وجود عناصر وعلاقات بينها دون أن يستلزم أن تؤدي العلاقات الى وحدات أكبر، وإنما يمكن ان يقوم النظام بين أفراد متوازية، أي ليس بعضهيا جزءا لبعض.

تتمثل علاقة النظام بالتركيب في اتساع مفهوم النظام عن التركيب، اذ يمثل التركيب صورة من صور النظام، ولذلك يستلزم البحث الحالي وجود وحدة كبرى ذات وحدات صغرى مترابطة للقول بوجود التركيب. على أن في الدرس اللغوي تفريقا أخر بين النظام و التركيب، وهو مانجده عند فيرث الذي ركز كثيرا على الفرق المتوازي بين التركيب والنظام. فقد التفت إلى ضرورة تعامل التحليل النحو مع النصوص بتحديد كل من التراكيب والأنظمة. ويعني ذلك أن الفرق بين النظام والتركيب عند "فيرث" يعد نوعا ما من تطوير العلاقات الأفقية

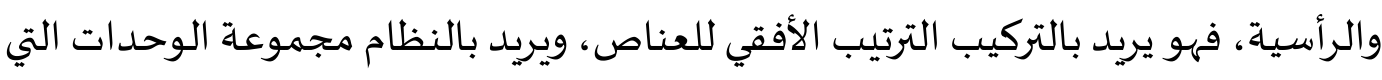
يندرج ضمنها عنصر ما. 
تركيب المعجم وتصنيف الدلالات

يجب ان يسجل البحث عن تركيب المعجم أو بنيته أول ما يسجل ورود نوعين من التركيب المعجمي، يتمثل أولهما في التركيب الخاص بمداخله، كما يتمثل الثاني في التركيب العام للمعجم كله، فإن المعجمي يبدأ بتركيب المفردات معا في مدخل، ثم يثني بتركيب المداخل معا في معجم عم وذلك على النحو التالي

- التركيب الخاص بالمدخل : يراد باه ترتيب المفردات التي ترد تحت كل مدخل من مداخل المعجم. ويرد المدخل من عمل المعجميين أنفسهم، إذ يضعون لمعجم كل لغة ما يروناه من

$$
\text { المداخل، ثم يدرجون بكل مدخل ما يرون دخوله من مفردات اللغة }
$$

- التركيب العام للمعجم : هو ذلك التركيب العام الذي يتم فياء تركيب المداخل في معجم واحد بعد أن تم جمع مفردات كل مدخل في مدخلها الخاص بها. وتسعى شتى النظريات المعجمية للوقوف على بناء المعجم اللغوي بعامة، أي بناء المداخل من مفردات، وبناء المعجم العام من مداخل. إنهم يحاولون تحديد بنية المعجم على نحو شامل واضح

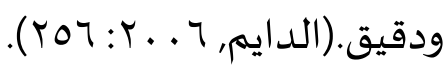

استقصى اللغويون مختلف العلاقات الدلالية وصنفوها وفق معايير مختلفة، كما وقفوا مع الرموز اللغوية التي تؤدي هذه الدلالات. وقد انطلقوا في ذلك من إدراك أهمية العلاقات، وربما تقدمها على الوحدات نفسها، يقول بعضهم في هذه الأهمية : إن الوحدات

$$
\text { الموذج التصبية للغة هي تلك العلاقات التي تعبر عنها الأصيوات والحروف والمعاني. }
$$

من أبرز ما صنفوه من علاقات دلالية بين الوحدات اللغوية تتصل بموضوعنا على نحو ما تصنيف سوسير الذي يمكن ان يكون السمة المميزة للغويات القرن العشرين، حيث يصنف العلاقات الى رأسية سماها اقترانية وأفقية. ويمثل تصنيف العلاقات هذا بيانا لكل من ()علاقات التركيب أو العلاقات الأفقية هي العلاقات التي تربط بين عناصر التركيب القائمة فعلا في التركيب لتكوين هذا التركيب. r) علاقات النظام أو العلاقات الرأسية هي تلك العلاقات التي تجمع أفرادا ما تحت نظام واحد، كتلك العلاقات التي بين الوحدات 
الصالحة للتناوب على الموقع النحوي الواحد. وهذه العلاقات لاتنتج تركيبا، اذ لا تركب

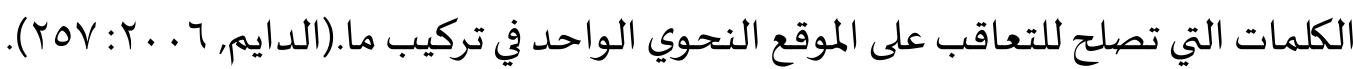
نموذج التصنيف وفق معيار اللغة والواقع

إن اللغويين العرب قاموا بمراعاة بعض العلاقات الدلالية في بنائهم للمعجم دون بعض آخر، فهم يبنونه على أساس العلاقة الدلالية بين المفردات المتحدة في الجذر، ولا يراعون دلالات أخرى كالترادف والتضاد والانضواء. () العلاقات الدلالية التي يسمح بها الجذر أو يكشف عنها : (الاشتراك الجذري - الاشتراك اللفظي - الاشتراك الدلالي الاشتراك الدلالي - الاشتراك الوظيفي). r) العلاقات الدلالية التي لا يسمح بها الجذر أو لا يكشف عنها : ( الترادف والتضاد والانضواء) يرجح تفسير موقف المعجم المزدوج من الدلالات المختلفة في رؤية البحث الى انه قد رأى ورود العلاقات الدلالية التي بين المفردات على نوعين متمايزين، هما : ( ) العلاقات الدلالية اللغوية : هي ما كان من العلاقات ذا ارتباط باللغة، وليس مقتصرا على الواقع. ويتحقق ذلك فهي علاقة الاشتراك الجذري، علاقة الاشتراك اللفظي، وعلاقة الاشتراك الدلالي. r) العلاقات الدلالية الواقعية : هي تلك العلاقات التي تقوم بين مدلولات الكلمات دون ان نجد لذلك وجودا في اللغة او مرجعا لغويا لها، وذلك في علاقة الترادف وعلاقة التضاد وعلاقة الانضواء.(الدايم, 7 . . ؟ ع ب؟).

\section{منهج البحث}

تتركز عملية هذا الحث على أساس البحث المكتبي، يعني نوعا من أنواع البحث العلمي الذي يحدد عملية بحثه إلى المواد الوثائق والكتب في المكتبة ولا يحتاج إلى البحث الميداني (Mcmillan, 2011:78) وصف لظواهر أو الأشياء المعينة في جمع الحقائق والمعلومات والملاحظات عنها ووصف الظروف الخاصة بها وتقدير حالتها كما توجد عليه في الواقع. المنهج الوصفي هو يهدف إلى وصف لظواهر أو الأشياء المعينة في جمع الحقائق والمعلومات (Moleong, والملاحظات عنها ووصف الظروف الخاصة بها وتقدير حالها كما توجد عليه في الواقع. 
2002:11عند أريكونتو المنهج الوصفي هو البيانات التي تتكون من الكلمات والتعبيرات والمعلومات التي لها الترابط والتلازم وثيق الصلة بوقائع الظاهرة حين وقوع البحث ثم حللها الباحث للوصيول إلى تنيجة البحث.(Arikunto, 1998:309 ) وأما دراسة مكتبية هي بحث بعتمد على البيانات والمعلومات المتوفرة في الكتب والدرويات والمراجع العامة, حيث يبدأ بالقراءة والإطلاع ثم يبدأ بكتابة بحثه مستندا إلى تلك المصادر. وهذا البحث يتم إجراءه من على المكتب دون الحاجة للنزول إلى الميدان لجمع ملاحظات. وأما المدخل في هذا البحث هو المدخل الكيفي، لأن البحث الكيفي بأنه منهجية البحث في العلوم تركز على وصف الظواهر وصفا دقيقيا وعلى الفهم الأعمق لها (د غباري،

. (r):

أما بيانات المتعلقة بهذا البحث التي يتم جمعها من المصادر الأساسية يعني : كتاب

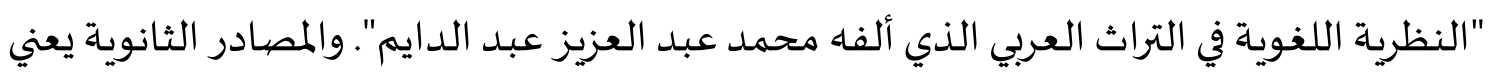

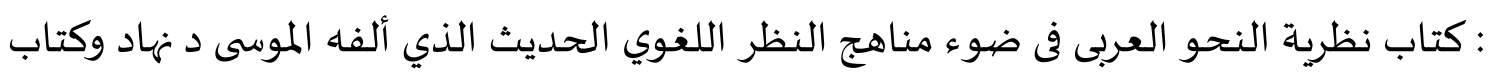

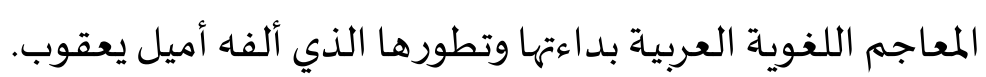

انطلاقا مما سبق يقوم الباحثون بجمع المعلومات التي تتعلق بالمسائل وهي بقراءة المراجع التي فهها مسائل مبحوثة. أما معطيات البحث المستخدمة فتنقسم إلى المصيادر الرئيسية والثانوية.

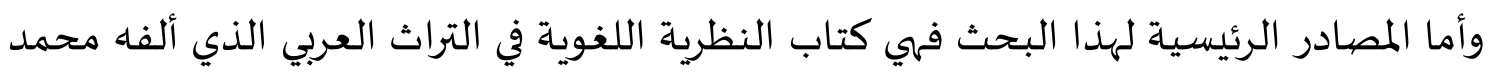
عبد العزيز عبد الدايم، والمصادر الثانوية فهي كتاب نظرية النحو العربى في ضوء مناهج النظر اللغوي الحديث وكتاب المعاجم اللغوية العربية بداءتها وتطورها وغير ذلك من الكتب المتعلقة بالبحث.

وينتهج الباحثون بتحليل البيانات باتباع الطريقة الأتية منها : جمع المعلومات المتعلقة

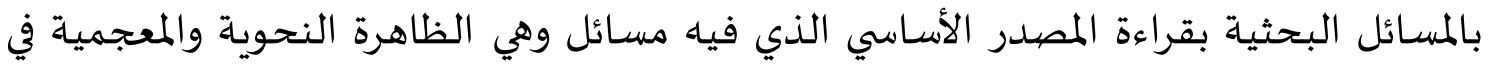
نظريات اللغوية. وتقسيم المعلومات إلى المصادر الرئيسية والثانوية. فالمصادر الرئيسية لهذا البحث

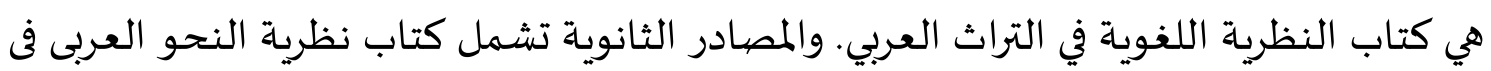
ضوء مناهج النظر اللغوي الحديث وكتاب المعاجم اللغوية العربية بداءتها وتطورها وغير ذلك من

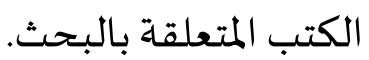




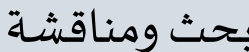

\section{نظريات الجمع والتصنيف المعجميين والتحليل الدلالي

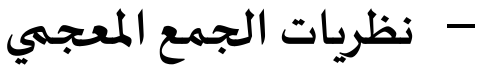

أول ما يثار بخصوص جمع العربية فرضية الخاص والعام، إذ يبادر كثير من الباحثين المى نفي وعي اللغويين العرب بالمستوى اللغوي الذي تنقسم باء اللغة الى لغة مشتركة وأخرى خاصة، يقول بعض الدارسين عن عمل اللغويين العرب : كان ينبغي للعلماء ألا يخلطوا بين اللغة ورسيلتها في الرواية أو الدراسـة، لكنهم لم يتنبهوا لهذا التحرز، فخلطوا بين اللغات المتعددة. والحقيقة أن اضطراب اللغويين وخلطهم بين اللغة المشتركة واللغة الخاصة أمر غير قائم. وأن كل ما يمكن أن يؤخذ على اللغويين العرب هو عدم تخصيص مباحث للغة المشتركة وأخرى خاصة باللغة الخاصةة، وهو أمر شائع في درسنا اللغوي الذي لم يفصل بين العلوم نفسها، فأورد بين دفتي كتاب واحد علوما شتى كالأصيوات والصرف والنحو. لقد وضع المعجميون العرب ثلاث دوائر متداخلة للعربية أصغرها دائرة العربية المستعملة، وهو مركز دائرة أكبر للعبية المقبولة تجمع دائرة العربية المستعملة مع هامش حولها للغة المهملة، وهي تلك اللغـة التي لم يتواضع عليها العرب، فلم ترد مستعملة عندهم، وهي غير مخالفة للغة المستعملة إلا في عدم تواضع العرب عليها. أي أن اللغة العربية المقبولة نوعان : أحدهما استعمله العرب، والثاني كان يمكن أن يستعملوه، إذ ليس فيها ما يمنع من

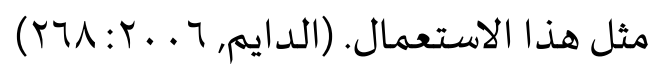

$$
\text { - - - نظريات التصنيف المعجمي }
$$

يقوم المعجميون العرب بتصنيف المادة التي يدرجونها في دائرة الغربية المستعملة التي

ليست محظورة صوتيا ولامهملة استعمالا فلا يتركونها ضبرا واحدا، بل يصنفونها بشكل أكثر تعقيدا وتركيبا على أسس لغوية عامة، إذ يصنفونها على النحو التالي : ( ) يرفضون بعض ما جمعوا من المادة اللغوية ويردونه بسبب من التصحيف والتحريف واللحن والخطأ والغلط والسهة، فيتشكل بهذا هامش للغة المردودة غير المقبولة يمثل هامشا خارجيا يحيط بدائرة اللغة المستعملة. r) يجعلون غير المردود من اللغة المستعملة على صنفين، هما : اللغة الخاصية بالشعر او باللهجة واللغة المشتركة، وبجعلون اللغة الخاصية هامشا داخليا للغة 
المستعملة فيلي بذلك هامش اللغـة المردودة، كما يبقى هذا الهامش خارج دائرة اللغة المشتركة، إذ يجيط بها من الخارج. r) يصنفون اللغة المشتركة بدورها الى شاذة ومطردة. وتمثل اللغة الشاذة هامشا تاليا لهامش اللغة الخاصة، ويكون هذا الهامش داخليا بالنسبة لدائرة اللغة المشتركة، كما يكون خارجيا بالنسبة لدائرة اللغة المطردة، إذ يحيط بها على

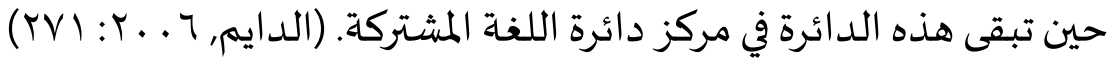
والحقيقة أن مفاهيم الجمع المعجمي والتصنيف المعجمي يمكن أن تصور معا بالرسم التالي الذي يبين الخطوات التي اتبعها اللغوييون العرب في معالجتهم المعجمية للغة العربية مثلما اتبعوها في معالجتهم الصرفية والنحوية :

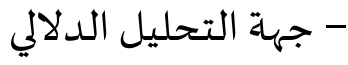
وذكر عبد الدايم أن اللغويين في التراث العربي قد استخدموا عدة مناهج في تحليلهم الدلالي مع كل صور الاتفاق اللفظي.(الدايم, T . . : YVV). وتلك المناهج هي : - الاشتراك الدلالي وهو المنهج الذي يفترض أن اللفظ واحد تشترك فية كلمات مختلفة ذوات دلالات متعددة، كأن تشترك في لفظ"أن" الحرف الناسخ والفعل الماضي "أن" - الإشتراك اللفظي وهو المنهج الذي يفترض أن الألفاظ متعددة قد اخذت شكليا لفظا واحدا ككلمة "عين " التي تكون للباصرة ولعين الماء... الخ(عمر, 910 1: 107). - الاشتقاق القياسي هو الذي يكون بين الكلمات المأخوذ بعضها من بعض عن طربق تغيير صرفي محدد بينه الصرفيون بالميزان الصرفي كاشتقاق اسم الفاعل من الثلاثي على وزن فاعل، ومن غير الثلاثي على وزن مضارعاه مع قلب حرف المضارعة ميما مضمومة وكسر ما قبل الآخر، واشتقاق اسم المفعول من الثلاثي على وزن مفعول ...

- - الاشتقاق السماعي هو الإشتقاق الذي قام باء ابن فارس حين مد مظلة القياس لتشمل ما لا يملك طريقا قياسية للاشتقاق مثل جمعاه تلك الكلمات التي لا تشترك إلا في الجذر، أي ذوات صلة جذر فحسب، دون ان تكون مأخوذة بعضها من بعض. - الاشتقاق الكبير هو منهج يرصد العلاقة التي تكون بين الجذر وتقليباته. ويعرف من هذا المنهج، أن الاتفاق بين الالفاظ بسبب اتفاقها في المادة لغوية دون أوزاهها، إذ الفرق 
في ترتيب الجذر الذي هو هيئة الجذر دون مادته أو أصوله. مثل : (ق و ل)، (و ل ق)، (و ق ل) و (ل ق و) وتقاليبها الستة بمعنى الخفة والسرعة. - الاشتقاق الأكبر : وقد طبقه ابن جني كذلك تحت عنوان "تصاقب الألفاظ لتصاقب

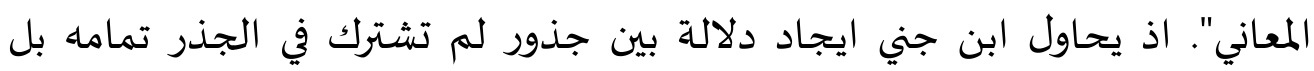
اشتركت فقط في جزء كبير من الجذر دون بقيته، مثل "أز" و"هز" فالهمزة أخت الهاء لهاء

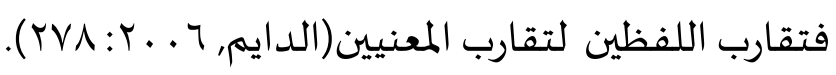

\section{نظريات التركيب المعجمي في النظرية اللغوية الغربية}

يلزم البحث استعراض موقف الدرس اللغوي الغربي من التركيب المعجمي قبل مراجعة

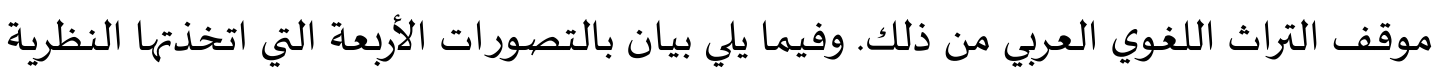
اللغوية المعاصرة بخصوص التركيب المعجمي للغة. يمكن الوقوف على أربع تصورات مختلفة

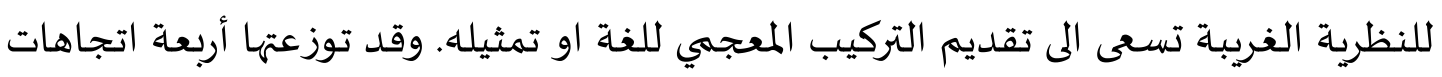

$$
\text { رئيسية، وهي ترد على ما يلي }
$$

- نموذج تقديم تركيب معجمي من خلال العلاقات الدلالية

وينص بعض اللغوييون على كون الوقوف على العلاقات الدلالية وتصنيفها يمثل بناء للمعجم، يقول : الهدف الرئيسي من علم الدلالة المعجمي بناء نموذج لتركيب المعجمه

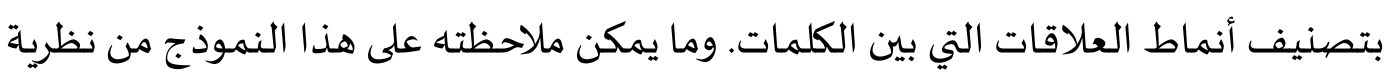

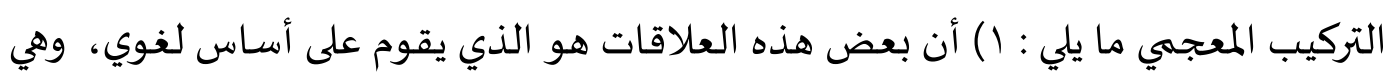

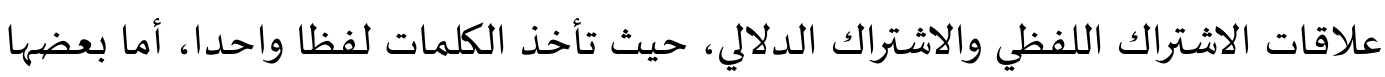
الآخر فهو يقوم بين مدلولات المفردات وفق الواقع لا وفق اللغة، فليس في الالفاظ اللغوية ما

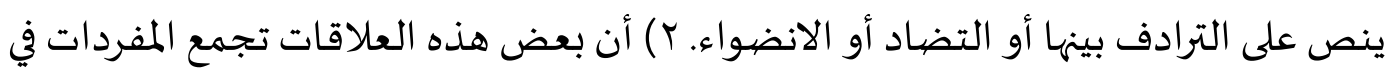

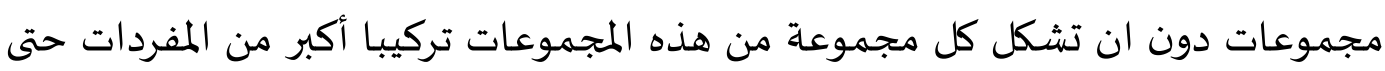

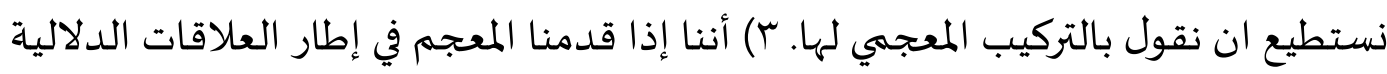
المذكورة، أي في إطار علاقات الترادف والتضاد والانضواء والاشتراك اللفظي والاشتراك

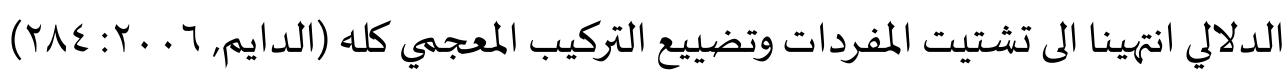


- - م-موذج تقديم تركيب معجمي من خلال معالجة المكونات الدلالية ويمثل تحليل مكونات الكلمة الدلالية جرء بارزا من العمل المفصل في تركيب المعجم الذي بذل في السنوات الأخيرة. وتتحرك نظرية المكونات الدلالية بين الوحدة المعجمية المفردة والمكونات الدلالية التي ركبت معا لتكوين هذه الوحدة الدلالية الاكبر. - - نموذج تقديم تركيب معجمي من خلال الحقول المعجمية

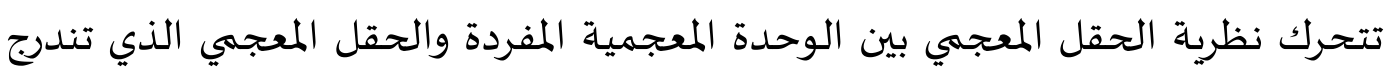
تحته هذه الوحدات المعجمية المفردة، ويتكون الحقل من مجموع هذه الوحدات والعلاقات التي تجمع بينها لتندرج تحت هذا الحقل إذا الحقل، هو التنظيم الذي يمكن أن تدرج فهها كلمات اللغة ليمكن فهمها على نحو دقيق. - - نموذج تقديم تركيب معجمي من خلال معالجة التركيب المعجمي لجمل اللغة ويعنى ذلك أن البحث عن بنية معجمية في النظرية اللغوية الغربية قد اتخذ أربع طرق تبحث اثنتان منهما عن الدلالة في علاقاتها الأفقية، وهما نظريتا العلاقات الدلالية والتركيب المعجمي للجملة على حين تبحث الأخريان عن الدلالة رأسيا بربط الوحدات المعجمية بما تحتها من مكونات أو سمات دلالية أو بما فوقها من حقول معجمية.

\section{نظريات التركيب المعجي في النظرية اللغوية العربية}

تتمثل تصورات التركيب المعجمي العام فيما استطاع البحث ان يقف عليه في أربعة نماذج يتصل اثنان منهما بالجذر، ويتصل الآخران بالسلسلة على ماسيرد بيانه فيما يلي :

$$
\text { - - نظرية الجذر المفرد (جذر الإشتقاق الصغير) - }
$$

يعد هذا النوع من الجذر أكثر النطريات استخداما في تمثيل التركيب المعجمي للعربية. ولكي نوضج كيف يقدم مفهوم الجذر هذا أصول نظرية واضحة للتركيب المعجمي الى ما يلي : 1) الجذر يمثل وحدة وسطى بين المعجم كله والكلمات، اذ يمثل تجميعا لمفردات اللغة، فلا تستقل كل كلمة بمدخل معجمي. Y) العلاقة الدلالية بين مفردات الجذر تنبني على أساس لغوي إذ ترجع الى اشتراك في الأصول. r) هذه العلاقات الدلالية التي يثبتها الجذر وينبني عليها

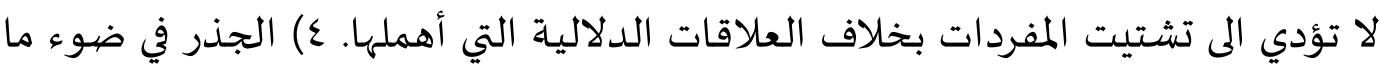


سبق يحقق أركان التركيب الثلاث، فهو الوحدة النركيبية الكبرى. 0) إهمال الجذر لبعض العلاقات الدلالية كالترادف والتضياد والانضيواء. 7) مفهوم الجذر لم يقم على مجرد العلاقة الأسـاسية له، وهي علاقة الكلمات التي تندرج تحتـه بسبب الاتفاق في الجذر. أما النوع الثاني من كلمات الجذر الواحد التي لا تجمعها صلة اشتقاق أصغر فقد اتخذ المعجم العربي له صورتين تمثلان نموذجين مختلفتين لتركيب المعجم، ما يلي : ( ) نموذج التركيب الشكلي للجذر (النموذج الدلالي الجزئي للجذر). r) نموذج التركيب الشكلي الدلالي للجذر (النموذج الدلالي الكلي للجذر)(الدايم, ج ـ . r: بوץ). - السلسلة المعجمية او سلسلة الجذور (جذر التقليبات)، نموذجان :

تقوم السلسلة المعجمية في مجموعة الجذور التي تمثل تقليبات لأصول واحدة، وهي وحدة وسيطة بين المعجم والجذر، فإن الجذر اذا كان يجمع عددا من المفردات تحته فإن السلسلة تزيد بجمع عدد من الجذور تحتها. وفيما يلي بيان لنموذجي السلسلة التي ترد كما يرد الجذر سلسلة شكلية فقط وسلسلة شكلية دلالية، وذلك على التفصيل التالي : ( ) نموذج السلسلة الشكلية أو الصوتية للجذور. ب) نموذج السلسلة الدلالية للجذور (جذر

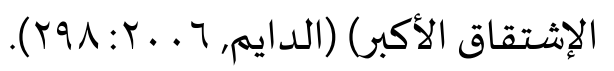

(خلاصة)

- المعجمية تحتوي مجموعة من مفردات اللغة مرتبة ترتيبا أبجديا، أو في نظام أخر محدد مع شرح معانها، وعادة ما يذكر المعلومات الخاصة بها في اللغة نفسها، أو في لغات أخرى. وأهميتها منها : المحافظة على سلامة اللغة، جعل اللغة قادرة على مواكبة العلوم والفنون، الكشف عن معاني الألفاظ المجهولة والغامضية، معرفة ظواهر اللغوية كالمشترك اللفظي والأضيداد، معرفة أصل اللفظ واستقاقاته، معرفة كون اللفظة عامية أو فاصحة. - النظرية المعجمية تقوم في عدد من الإجراءات الضرورية لإنجاز معجم اللغة تبدأ هذه الإجراءات بالجمع المعجمي اللازم لمفردات اللغة وتراكيبها واستعمالات كل من المفردات والتراكيب، ويتبع ذلك إجراءا الوصف والتصنيف للمادة المجموعة،، وتقديم التركيب أو 
- - نظريات التصنيف المعجمي بتصنيف المادة التي يدرجونها في دائرة العربية المستعملة التي

ليست محظورة صوتيا ولا مهملة استعمالا. وتتمثل تصورات التركيب المعجمي العام فيما استطاع البحث ان يقف عليه في أربعة نماذج يتصل اثنان منهما بالجذر، ويتصل الآخران

بالسلسلة.

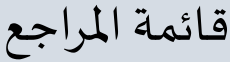

J. H. Mcmillan. (2011). Research in Education: A Conceptual Introduction. New York Longman.

Moleong, L. (2002). Metode Penelitian Kualitatif,. Bandung: Remaja Rosdakarya.

Suharsimi Arikunto. (1998). Prosedur Penilaian Suatu Pendekatan Praktek. Jakarta:

Rineka Cipta.

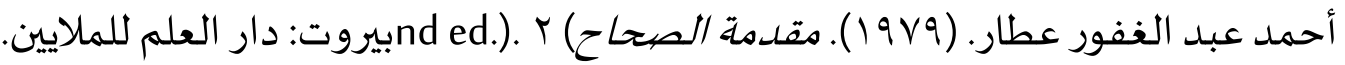

الدايه, م.ع. ا.ع. (7 . . r). النظرية اللغوية في التراث العربي. القاهرة: دار السلام.

الغني، ي. ع. (1991 ). معجمر المعاجم العربية. بيروت: دار الجيل.

تائر أحمد غباري، يوسف عبد القادر أبو شندي، خالد محمد أبو شعيرة. (9 . . ب). البحث النوعي في التوبية وعلوم النفس. عمان: مكتبة المجتماع العربي للنشر والتوزيع. عمر, أ. م. (910 (1)).علم الدلالة. القاهرة: عالم الكتب. مجد البرازي. (919 (19). مشككلات اللغة العببية المعاصرة. عمان: مكتبة الرسالة. منظور, ا. (1907 ). اللسان. بيروت: دار صيادر ودار بيروت. وافي, ع.ع. ا. (19VY) ). فقه اللغة. القاهرة: دار النهضة مصر للطباعة والنشر. يعقوب, أ. (919V (1). المعاجم اللغوية العببية بلداءها وتطورها. بيروت.لبنان: دار العلم للملايين. ابن هشـام، (1919) (1). مغنى اللبيب عن كتب الاعارب ,تحقيق د. مازن المبارك وعلي حمد الله, دمشق

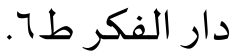

Please cite this article as Muhammad Qodri. (2019). Lexical Phenomenon in Linguistics Theories. Izdihār : Journal of Arabic Language Teaching, Linguistics, and Literature, 2(1), 1-16. DOI: 
أحمد مختار عمر، (1910). البحث اللغوي عند العرب مع دراسة لقضية التأثير والتأثر، القاهرة : عالم الكتب. عبد القادر أبو شريفة، (919 19). علم الدلالة والمعجم العـبي، عمان : دار الفكر. محمد عبد العزيز عبد الدايم، (7 . . ؟). النظرية اللغوية في التراث العربي، القاهرة : دار السالم. 